\title{
Lista de Pareceristas
}

\author{
Alexandre Plautz \\ Alfio Brandenburg \\ Amanda Stinghen \\ Anna Carletti \\ Antônio Fernandes Nascimento Junior \\ Beatriz Klimeck Gouvêa Gama \\ Beatriz Rangel Thurler Amorim \\ Bruna Angotti \\ Eduardo da Silva \\ Eduardo Oliveira de Almeida \\ Guilherme Abilhoa \\ Jairo Antonio Bosa \\ Judit Gomes da Silva \\ Juliana Abonizio \\ Kamille Brescansin Mattar \\ Katiano Miguel Cruz \\ Lívia Boechenstein \\ Lucas Buosi \\ Luciano Celso Brandão Guerreiro Barbosa \\ Mabile Caetano Cazela \\ Marcela Cockell \\ Maria Izabel Machado \\ Marlene Tamanini \\ Miriam Adelman \\ Nashyelli Salazar Flores \\ Patricia Guerino \\ Rafael da Rocha Massuia \\ Rebeca Gontijo \\ Rodrigo Graça \\ Sara Guerreiro Parada \\ Thais Madeira \\ Thays Monticelli
}

